\title{
RECREACIÓN COMUNITARIA DEL CAMPESINADO EN LA INTERFASE SOCIO - JURÍDICA: EL CASO DEL PARAJE LIMITAS EN EL EX - INGENIO LAS PALMAS (CHACO, ARGENTINA)
}

\section{COMMUNITY RECREATION- CAMPESINADO IN -JURÍDICA PARTNER INTERFACE: THE CASE OF THE EX LIMITAS SPOT -INGENIO LAS PALMAS (CHACO, ARGENTINA).}

Diego Domínguez ${ }^{1}$

Pablo Barbetta ${ }^{2}$

\section{Resumén:}

La consolidación del modelo del agronegocio en Argentina trajo consigo mayores niveles de concentración de la tierra y tendió, de diversos modos, a profundizar procesos de exclusión y expulsión de familias del campo. Al mismo tiempo, supuso un aumento de la conflictualidad en el agro argentino en torno a la propiedad de la tierra y el uso de los bienes naturales. El alcance de este proceso se puede observar en la creciente conflictualidad territorial en áreas rurales puesto que allí se activa la presencia de una intencionalidad campesina que reivindica un modo de vida y su derecho a la tierra. La provincia del Chaco no está excluida de dichos procesos. En este contexto de vitalidad social y política del campesinado chaqueño nos interesa analizar y comprender el proceso de "invención" de una figura de tenencia comunitaria del territorio en el Paraje Limitas, departamento de Bermejo, en el marco del proceso organizativo de la Unión de Pequeños Productores del Chaco (UNPEPROCH). Más precisamente, nos proponemos indagar los procesos que confluyeron en esta figura que realiza el derecho a la tierra en una interfase socio jurídica, en donde intervienen las poblaciones pre existentes, trabajadores sin tierra, el Estado y actores del agronegocio.

Palabras clave: campesinado - tenencia comunitaria - interfase socio - jurídica.

\section{Abstract:}

The consolidation model of agribusiness in Argentina resulted in higher levels of land concentration and tended in various ways to deepen processes of exclusion and expulsion of rural families. At the same time, meant an increase in the Argentine agro conflictivity around land ownership and use of natural resources. The scope of this process can be seen in the growing territorial conflictivity in rural areas because there the presence of intentionality that campesinas a peasant way of life and their right to land on. The province of Chaco is not excluded from these processes. In this context of social and political vitality of the Chaco peasantry we want to analyze and understand the process of "invention" of a figure of communal tenure of land in the Place Limitas, Bermejo department, under the organizational process of the Union of small producers Chaco (UNPEPROCH). More precisely, we propose to investigate the processes which will converge in this

\footnotetext{
${ }^{1}$ Prof. Dr. Investigador del Instituto de Investigaciones Gino Germani, Fac. de Cs. Sociales, Universidad de Buenos Aires. E-mail: didominguez1@yahoo.com

2 Prof. Dr. Investigador del Instituto de Investigaciones Gino Germani, Fac. de Cs. Sociales, Universidad de Buenos Aires. E-mail: pablo_barbetta@yahoo.com.ar
} 
figure performing the right to land in a legal partner interface, where the pre-existing, landless workers, the state and agribusiness actors involved populations.

Keywords: Campesinado -Interface -jurídica partner community tenure

\section{INTRODUCCIÓN}

La consolidación del modelo del agronegocio en Argentina trajo consigo mayores niveles de concentración de la tierra y tendió, de diversos modos, a profundizar procesos de exclusión y expulsión de familias del campo. Al mismo tiempo, supuso un aumento de la conflictualidad en el agro argentino en torno a la propiedad de la tierra y el uso de los bienes naturales (agua, bosques) como consecuencia de situaciones problemáticas de tenencia de la tierra; expansión de los problemas ambientales y casos de contaminación rural y emergencia de hechos de violencia en el campo que se ponen en juego, por ejemplo, en los desalojos y/o cercamientos de poblaciones indígenas y campesinas (GER, 2004; GEPCYD, 2009).

En este contexto, el paso del denominado modelo de desarrollo agropecuario agroindustrial hacia la hegemonía de los agronegocios implica que la reproducción de los sectores subordinados (trabajadores, medianos y pequeños productores, campesinos, etc.) dejara de estar implícita en la lógica capitalista (Giarracca y Teubal, 2008) y por ende, coadyuva a reforzar las teorizaciones descampesinistas como consecuencia del desarrollo del capitalismo en el agro. Sin embargo, frente a estos condicionamientos estructurales, el caso del campesinado en algunas provincias argentinas muestra en sus resistencias y estrategias de re-producción, la vitalidad de un sujeto social. Ya desde finales de la década de 1980 se registran experiencias de acción colectiva de poblaciones que se auto-reconocen como campesinas. Se trata de una emergencia que se expresa en dos dimensiones: en la arena pública fundamentalmente en referencia a conflictos o demandas por el acceso y las formas de tenencia de la tierra, y en la factualización de múltiples experiencias que operan decenas de organizaciones y que se orientan a la agroecología, mercados locales y comercio justo, rescate de los conocimientos ancestrales, asesoramiento legal y técnico, educación rural formalizada y formación política, gestión comunitaria de proyectos productivos, entre otros espacios sociales para la promoción de una "territorialidad campesina" (Barbetta, Domínguez y Sabatino, 2012). El alcance de este proceso se puede observar en la creciente conflictualidad territorial en áreas rurales puesto que allí se activa la presencia de una intencionalidad campesina que reivindica un modo de vida. Según estiman investigaciones oficiales del año 2013 se 
registraron 857 problemas de tierra en todo el país, donde se disputan unas 9.293.233,80 hectáreas, implicando a 63.843 agricultores familiares (Gigena, 2013).

En la provincia del Chaco se constata también, por un lado, procesos de recampesinización que van desde el vuelco de ex trabajadores rurales a la producción agropecuaria hasta el cambio de vida que atraviesan ex colonos algodoneros que comenzaron a diversificarse en función de la producción de alimentos para el autoabasto y para la comercialización de los excedentes. Asimismo, se observa la consolidación de instancias organizativas propias del campesinado, cuya heterogeneidad pone en evidencia la riqueza del proceso, por ejemplo en la Mesa de Organizaciones de Pequeños Productores del Chaco, surgida en el año 2006, y la posterior conformación de la Asamblea Campesina e Indígena del Norte (ACINA) que sintetiza un espacio de encuentro regional de organizaciones de las provincias del noreste argentino.

En este contexto de vitalidad social y política del campesinado chaqueño nos interesa analizar y comprender el proceso de "invención" de una figura de tenencia comunitaria del territorio en el Paraje Limitas, departamento de Bermejo, en el marco del proceso organizativo de la Unión de Pequeños Productores del Chaco (UNPEPROCH). Más precisamente, nos proponemos indagar los procesos que confluyeron en esta figura que realiza el derecho a la tierra en una interfase socio jurídica, en donde intervienen las poblaciones pre existentes, trabajadores sin tierra, el Estado y actores del agronegocio. En ella se compone una situación de interlegalidad, definida como la superposición, articulación e interpenetración de varios espacios jurídicos mezclados (Santos; 2001). Para nuestro caso de estudio, la presencia de una interfase con distintos actores en disputa tanto por el espacio geográfico como por el derecho a la tierra, hace a la singularidad de esta situación de interlegalidad. En efecto, más que superposición, articulación o interpenetración de espacios jurídicos, debemos hablar de una situación de tensión en torno a ellos, ya que se pone en juego el reconocimiento del campesinado como sujeto de derecho y con derecho al acceso de la propiedad de la tierra, y en base a ello, un proceso de distribución de los medios de producción (los bienes naturales).

Desde el punto de vista metodológico, este trabajo se sustenta a partir de entrevistas en profundidad individuales a dirigentes y miembros de la organización como así también entrevistas colectivas realizadas en el marco del Proyecto de Voluntariado Universitario "Cartografía social y comunidades campesinas: fortalecimiento de procesos participativos desde la construcción colectiva del territorio", 2009-2011. Las frases entrecomilladas y en cursivas corresponden a las voces de los actores, y sin cursiva a citas de estudios y análisis. 
El trabajo está dividido en tres apartados. En el primero de ellos, abordamos los principales sucesos históricos en la conformación de Limitas. En el segundo apartado, analizamos los procesos de configuración socio-económica y política de la actual población de Limitas, y a partir de ello la elaboración de una experiencia de control comunitario de la tierra en el marco de una situación que definimos como de interlegalidad. Finalmente, una breve conclusión.

\section{HISTORIA DE LAS PALMAS Y DEL CONTEXTO CHAQUEÑO}

El patrón de acumulación capitalista en lo que sería la geografía de la actual provincia del Chaco se reconfiguró, desde las últimas décadas del Siglo XIX, a partir de la explotación forestal, la ganadería y sobre todo, el cultivo de algodón, principal actividad de la provincia, que combinaba la formación de grandes explotaciones con el asentamiento de productores directos en el marco de políticas de colonización (Adámoli et al 2010, Mari; 2009, Dal Pont y Ordoqui; 2008). En la década de 1960, con la "frontera cerrada", más del cincuenta por ciento de las explotaciones agropecuarias chaqueñas pertenecían a predios de entre 25 y 100 hectáreas, basadas en mayor medida en el trabajo familiar y en el cooperativismo. El cultivo de algodón era por entonces el gran dinamizador de la economía de la provincia, a la vez que un dispositivo de identificación de la población (Slutzky; 2011). Es a partir de la década de 1990 que se produjo la desestructuración de la matriz algodonera y consecuentemente la desarticulación de la convergencia de los actores imbricados en el complejo agroindustrial (GEPCyD, 2009). En estas nuevas condiciones se reabrió la "frontera", se recrudece el apremio empresarial sobre poblaciones campesinas e indígenas, vía cercamientos, arrinconamientos y desalojos. El avance del frente sojero, arrocero y maderero produjeron la ampliación de la frontera agraria y la valorización de la tierra, en un marco general de desacople de los productores campesinos algodoneros de la cadena agroindustrial y de retracción de la demanda de mano de obra rural. La nueva presión empresarial sobre la tierra se dio en condiciones de prescindencia con respecto a las poblaciones rurales (GEPCyD, 2009).

Sin embargo, en los últimos años de la década del 2000, el gobierno de la provincia de Chaco impulsó políticas de desarrollo rural a partir de la instrumentación de dos dispositivos jurídico-administrativos: consorcios de servicios rurales y fideicomisos. Según el gobierno provincial, con los primeros, creados por ley 6547 de 2010, se buscó instalar capacidades en las localidades rurales para dar respuesta a las demandas de infraestructura y producción, como puede ser la adquisición de maquinaria, entre aquellos sectores que no tienen escala o 
posibilidades de capitalización. El segundo dispositivo, forma parte de una estrategia general en la provincia de impulso a la producción e infraestructura por la vía de este tipo particular de mecanismos económicos de financiamiento y articulación institucional. El gobierno viene llevando a cabo, con mayor intensidad desde el año 2009, un conjunto variado de fideicomisos, de los cuales algunos se refieren al ámbito rural y la producción agropecuaria: producción ganadera, comercialización de carnes, producción forestal, respaldo financiero para crédito a productores, reactivación de industria frigorífica, creación de reservas naturales, etc.

Los procesos actuales de Limitas se vinculan estrechamente con las mencionadas transformaciones del agro chaqueño en general, pero especialmente con lo ocurrido en la zona de Las Palmas. El paraje Las Limitas, ubicado en el chaco húmedo, es parte de las tierras del ex ingenio Las Palmas, departamento de Bermejo, provincia de Chaco. Su importancia radica en que, según el Senado de la Nación:

LAS PALMAS, es un pueblo que constituye un hito para la Argentina por haber sido el primer espacio regional que contó con luz eléctrica, en 1885, evidenciando esfuerzo y anticipación. (...) La localidad de Las Palmas, ubicada a $68 \mathrm{~km}$, de Resistencia fue fundada el 10 de mayo de 1882, cuando los hermanos Ricardo y Carlos Hardy fundaron un ingenio azucarero llamado Las Palmas del Chaco Austral. El establecimiento de este ingenio reviste gran importancia histórica, para la provincia del Chaco y es a la vez un hito fundamental en nuestro país, ya que, como hecho inédito, una pequeña usina proporcionaba luz eléctrica al naciente pueblo, convirtiéndose en el primer alumbrado público de la Argentina. La concesión de 100 mil hectáreas de tierra, una superficie varias veces más grande ocupada y la instalación del primer ferrocarril de trocha angosta, dio lugar a la creación de un complejo industrial construido en medio del monte. (Expediente Número 1337/05, del Senado de la Nación, Secretaría Parlamentaria, Dirección Publicaciones)

De esta manera, la historia oficial del temprano avance de la frontera agropecuaria en el espacio geográfico que integra hoy a la provincia del Chaco, establece mojones paradigmáticos como puede ser la fundación del Ingenio Azucarero Las Palmas del Chaco Austral, sobre los márgenes Río Paraguay, cercano a la ciudad de Resistencia. El relato oficial de la provincia de Chaco sitúa este hecho como parte de un discurso modernizador y celebratorio del despliegue civilizatorio en la región. Como lo refleja la declaración del Senado, la fundación del Ingenio Las Palmas apuntó a poner su fundación en la voluntad de progreso de los hermanos irlandeses de apellido Hardy, responsables de haber instalado una red eléctrica y un puerto en medio de una naturaleza percibida como indomable. Estos "pioneros" iniciaron la producción de caña de azúcar en la zona desde el año de 1882 y la construcción de la fábrica de azúcar y alcohol, para lo cual 
contaron con "obreros de Buenos Aires y Corrientes" y con "la colaboración de los indios tobas que poblaban la región" (http://www.chapay.com.ar/).

Según recuerdan los ex jornaleros del Ingenio que hoy habitan Limitas, en tiempos del Ingenio, se traían cosecheros - "tropas" de diferentes provincias, incluso de países limítrofes. Muchos de ellos son descendientes de correntinos, formoseños, paraguayos, o chaqueños de otras zonas. Recuerdan que la zona estuvo muy poblada. Trabajaban cosechando caña de azúcar. Fuera de los períodos de zafra, muchas familias migraban a cosechar algodón a otras zonas de la provincia. A contrapelo del discuro oficial, según los relatos de los ex jornaleros, las condiciones de vida y trabajo en Las Palmas no eran satisfactorias. Además, recuerdan episodios de enormes privaciones y sacrificios, incluso en algunos casos mencionan coacciones directas sobre los trabajadores (reclutamiento forzosos, castigos por protestas, sanciones laborales, etc), durante y posteriormente a los Hardy, incluso cuando se lo privatizó en los años de 1970 hasta la decada de 1990 cuando ya se remató el Ingenio.

Las tierras de Las Palmas, incluidas las de Limitas, pasaron por diferentes situaciones. En un inicio, en el marco del proceso de colonización, algunos estudios históricos mencionan la presencia de comunidades Qom y de un establecimiento jesuita (Bergailo, 2002). Ya en 1882, cuando se intensificó la avanzada militar en la frontera del gran chaco, se registró la ocupación de esas tierras por los Hardy, quienes obtuvieron hacia principios de siglo los títulos de propiedad definitivos por más de 80.000 hectareas. Luego el Ingenio y sus tierras pasaron a manos de la Sociedad Anonima Las Palmas del Chaco Austral, bajo la presidencia de uno de los hermanos Hardy. Finalmente, en el año 1971, el Ingenio fue estatizado, y con ello pasaron unas 100 mil hectáreas al dominio del Estado. En el año de 1993, con la quiebra definitiva del Ingenio, las instalaciones y tierras de Las Palmas fueron a remate, contemplándose las tierras que pasaron a constituir el perímetro urbano de Las Palmas y La Leonesa. Finalmente, producto de diversos procesos, incluso de alta conflictualidad, la situación de las tierras se tornó extremadamente variable. O sea, si bien en general el encargado de su control ha sido el Organismo Nacional de Administración de Bienes del Estado $(\mathrm{ONABE})^{3}$, se sucedieron cesiones de tierras para diversos proyectos productivos, compra de tierras por parte de la provincia de Chaco, apropiaciones por parte de empresarios forestales y agropecuarios que comenzaron a explotar la zona, obtención de títulos de propiedad por parte de

\footnotetext{
${ }^{3}$ Desde agosto de 2012, el ONABE se pasó a llamar Agencia de Administración de Bienes del Estado.
} 
antiguos pobladores, entre otras situaciones de singulares características como aquella ocurrida en Limitas.

Con el cierre definitivo del Ingenio en 1993 se ensayaron distintas acciones desde el Estado en relación con los pobladores y las tierras, de lo que había pasado a ser entonces el ex Ingenio Las Palmas. En relación con las tierras desocupadas, algunas fueron rematadas a favor de privados, empresarios agropecuarios, otras permanecieron en el dominio público como tierras de la Nación. En relación con los ex trabajadores, que habitaban tierras del ex Ingenio, el interventor estatal implementa de manera generalizada la estrategia de ofrecer indemnizaciones para que abandonen las tierras que ocupaban.

Una parte significativa de los ex trabajadores rurales, frente a la perdida del empleo, rechazaron las indemnizaciónes, y decidieron no migrar, no abandonar el lugar en busca de otras aternativas asalariadas, sino que al contrario, optaron por permanecer en las tierras, asumiendo la puesta en produccion de las mismas, y resistendo en algunos casos intentos de desalojo.

Con este escenario, de creciente conflictualidad, las iniciativas estatales se diversificaron en relación con las familias que permanecían en la tierra, aunque, según los testimonios recogidos entre los habitantes actuales de Las Palmas, siguieron generando incertidumbre, y cuando no, riesgo cierto de desalojo o arrinconamiento productivo. En los primeros momentos, año 1994, el gobierno provincial impulsó la conformación de cooperativas con ex trabajadores transitorios del ex Ingenio para explotar tierras de Las Palmas ocupadas por grupos de ex trabajadores permanentes del Ingenio. Por otra parte, en ese mismo momento, a algunas familias se les ofreció la entrega de tierras. Mientras unas aceptaron desde un inicio, otras rechazaron la propuesta, pues no estaba claro cuanta superficie recibirían, y temían no poder acceder luego a una superficie apta para la producción. Los pobladores experimentaron como amenaza el hecho de acceder solamente a pequeñas parcelas para vivienda, sin que se contemplara el despliegue de actividades productivas, que ya estaban siendo realizadas por ellos mismos. En casos, como en el paraje El Palmar, los pobladores afirmaron que fueron beneficiados con entregas de tierras de hasta siete hectareas por familia, sumando unas 190 hectáreas a 28 familias. Entre las intervenciones estatales también cabe mencionar los casos de acoso policial con intención de desalojar a familias que decidieron no abandonar las tierras que fueran asignadas por el Ingenio Las Palmas a sus padres o abuelos en tanto "trabajadores permanentes". En los últimos años el Estado, tanto nacional como provincial, ha dado un giro a partir de políticas de desarrollo rural en la zona, implementando diversos dispositivos para "jerarquizar" la producción familiar. 
Paralelamente, en el marco del desmantelamiento del Ingenio Las Palmas, por un lado, diversos empresarios buscaron, vía legal o ilegal, explotar esas tierras, por el otro, surgieron iniciativas colectivas desde los pobladores, que habían decidido no abandonar las tierras. Las familias comenzaron un proceso novedoso de organización como campesinos y pequeños productores, definiendo sus propias respuestas comunes a la situación de desmantelamiento definitivo del Ingenio Las Palmas.

De esta manera, la confluencia de las diversas estrategias, de apropiación y dominio del espacio geográfico de Las Palmas por parte de sujetos sociales diversos, ha ido produciendo una creciente conflictualidad por la tierra. En un primer momento, la presión empresarial, sobre las tierras que ocupan las familias, se realizó a partir de la extracción de madera o zonas de pastaje de ganado bovino. En el momento actual la principal intervención empresarial está dada a partir de la producción arrocera. Estos empresarios, representados en la zona a partir de la Asociación de Productores de Arroz, utilizan tierras del ex Ingenio, en carácter de propietarios o arrendatarios, construyendo infraestructura necesaria para este monocultivo, como canales de riesgo por inundación, que extraen agua de los cursos naturales y la devuelven a los mismos con la carga de agroquímicos luego del ciclo productivo. Al mismo tiempo, intentan avanzar sobre tierras que ocupan familias campesinas. Es ahí donde se entablan los principales conflictos, aunque también existen denuncias y litigios por contaminación producida a partir de los arrozales.

Hemos registrado un conjunto de conflictos o tensiones, entre familias campesinas y empresarios o el mismo Estado, ocurridos en los parajes donde tiene presencia la UNPEPROCH. Durante los primeros años posteriores al cierre del Ingenio, la presión de los pobladores organizados hizo que el gobierno provincial comprara tierras para destinarlas a familias de ex trabajadores del Ingenio, redefinidos ahora por el Estado y por ellos mismos como "pequeños productores". La organización logró, mediante movilización y acampe frente a la legislatura provincial en la ciudad de Resistencia, que se otorguen tierras para las familias de los diferentes lotes de los parajes de Las Rosas, Limitas y el Palmar. Experiencia testigo de esto fue la sanción de la ley 4243/95 de la provincia, por la cual el gobierno del Chaco compró 753 hectáreas del lote 311, del ex Ingenio, para afectarlas especialmente a pequeños productores mediante el Instituto de Colonizacion Rural. En paralelo, comunidades indígenas comenzaron a reclamar su derecho constitucional a la tierra, y fueron logrando así títulos comunitarios de lo que había sido el Ingenio Las Palmas. 
A su vez, las tensiones y juicios por la tenencia de la tierra con empresarios se mantuvieron, con intentos de desalojo contra los pobladores. En estos casos la resistencia colectiva de hecho y vía derecho, llevada a cabo por comunidades campesinas e indígenas, hizo con que los habitantes consolidaran su presencia en aéreas, suficientemente extensas y biodiversas, con condiciones para realizar sus actividades productivas.

En esta línea, las poblaciones organizadas en la UNPEPROCH han elaborado experiencias singulares de control comunitario de las tierras y manejo colectivo de la producción. Entre otros, este es el caso del paraje Limitas.

\section{ACONTECIMIENTOS Y RECONFIGURACIONES SOCIO-JURÍDICAS EN LIMITAS}

Con el desguace del Ingenio Las Palmas, las personas que trabajaban allí y poblaban esas tierras, dejaron una condición asalariada con usufructo de pequeñas parcelas para vivienda, y pasaron a una situación incierta, sin trabajo y sin propiedad. Frente a este escenario las familias de Las Limitas adoptaron diversas respuestas. Según los testimonios recogidos, en algunos casos, se optó por el traslado definitivo, en general a las ciudades de La Leonesa o Las Palmas, o bien Resistencia. Mientras que otras familias del conjunto de ex jornaleros decidieron permanecer en las tierras que ocupaban ya sus padres y abuelos. La decisión de una parte de los habitantes de Limitas, de no migrar, se inscribió en el marco de un procesos de organización iniciado en el año de 1986, con apoyo de INCUPO (Instituto de Cultura Popular), y consolidado un año después en 1987, con la conformación del grupo de base de Limitas como parte de la UNPEPROCH (Unión de Pequeños Productores Chaqueños). Dos décadas después, en el año 2007, se sumaron al proceso de organización local y acceso a la tierra un grupo de familias provenientes del departamento de San Martín, integrantes también de la UNPEPROCH.

La organización campesina, de escala provincial, enraizada en grupos de base como el de Limitas, se postuló primeramente como herramienta para una reconversión productiva, en una provincia signada históricamente por la cadena algodonera, y a la vez, como vehículo de acceso a la tierra. Estas dos líneas de acción, en términos generales, dan cuenta de aquello que la UNPEPROCH postula como su objetivo central: "ayudar a que la gente no salga del campo". Para ello echa mano de múltiples estrategias, entre las cuales se halla la recampesinización por la vía de la obtención de tierras para poblaciones rurales proletarizadas o de agricultores profesionalizados que se empobrecieron con la crisis algodonera. 
La UNPEPROCH destaca haber puesto bajo control de familias rurales entre 18 y 20 mil hectáreas en distintos sitios de la provincia (GEPCYD, 2009). En este sentido sostenemos que el paraje Limitas forma parte de un conjunto más vasto de experiencias que desenvuelve la UNPEPROCH como parte de la regeneración de un campesinado chaqueño. En efecto, la organización impulsa en forma generalizada la formación de "Reservas" campesinas, entendidas como aquellas tierras adquiridas para que se instalen familias campesinas pero que se mantienen bajo la gestión de la organización. De hecho, las "Reservas" constituyen una de las principales estrategias con la que se busca territorializar la intencionalidad política de reivindicación del productor directo y su derecho a la tierra.

Según un informe de la Secretaria de Desarrollo Rural y Agricultura Familiar de la Nación, dependencia Chaco, el proceso de tenencia por el cual pasaron las tierras de Limitas es el siguiente:

Las tierras del paraje que pertenecieron al Ingenio, luego de su cierre pasaron a manos de la ONABE, que a su vez, en el año 2008 le transfiere la titularidad a la Comisión Nacional de Tierras para el Hábitat Social 'Padre Carlos Mujica'. Ese mismo año, dicho organismo comenzó un proceso de regularización de las Tierras, en acuerdo con la provincia y la organización a través de la figura del fideicomiso. La Comisión Nacional de Tierras para el Hábitat Social 'Padre Carlos Mujica' tiene a cargo los lotes 323, 324, 359, 360 y 278. Los restantes lotes ya se encuentran regularizados y destinados al asentamiento de las familias.

(Informe Limitas, Secretaria de Desarrollo Rural y Agricultura Familiar de la Nación, Chaco)

También indica el informe que la población de Limitas actualmente recibe apoyo gubernamental desde el INTA y desde los Consorcios de Servicios Rurales que realizan laboreos de suelos a los habitantes. En el documento se establece que el espacio geográfico de Limitas está compuesta por 8 lotes que suman 2972 hectáreas, donde viven 26 familias, que totalizan, según un censo realizado, 101 personas.

Para la dirigencia local y provincial de la UNPEPROCH, el proceso que se desenvuelve en la "Reserva de Limitas", constituye un caso emblemático. Para nosotros su importancia remite, por un lado, a que el proceso que conllevó a la conformación de la "Reserva de Limitas" contiene en sí dos acontecimientos (Badiou, 1999): uno que remite a las posiciones de sujeto y otro a la emergencia de la dimensión comunitaria tanto en la organización de la producción como en el manejo de los bienes naturales. A su vez, estos acontecimientos conllevan una determinada relación con el Estado configurando, para nosotros, un caso singular de interlegalidad en torno a la tenencia y uso de un territorio determinado. 


\section{Des-subjetivación y Re-existencia}

En Limitas, actualmente los pobladores reconocen que tanto los cambios que debieron enfrentar en torno de sus actividades productivas, como el hecho de asumir el derecho a la tierra, fueron la condición de posibilidad de su permanencia efectiva en el lugar. Si bien, entre los jornaleros del Ingenio se mantenía el hábito de cultivar mandioca, maíz, zapallo, y tener huertos y algún animal de granja, la actividad principal estaba asociada al trabajo en los cañaverales, o monocultivos de caña de azúcar. Recrearse como "pequeño productor" y "hacer por vos", según los relatos, implicó asumir una actitud diferente frente al trabajo y también adquirir otros conocimientos y capacidades prácticas. De similar forma ocurrió con las familias provenientes del departamento San Martín. Si bien no eran trabajadores asalariados, sino productores algodoneros, venían de trayectorias marcadas por desplazamientos permanentes por su condición de arrendatarios, o sin tierra, y por determinaciones externas para las decisiones productivas, por su posición subordinada ante el núcleo de poder del complejo agroindustrial algodonero. En algunos casos la tradición familiar o laboral sirvió como reservorio a ser reactualizado. En otros, la incorporación de cuestiones nuevas tuvo un peso mayor, pero en todos los casos hubo algún tipo de reorientación de las propias trayectorias de vida en el marco de una novedosa experiencia de organización. Los mismos protagonistas expresan, en sus historias de vida, la alquimia que debieron realizar con su propia existencia, destacando de forma diferente el rol jugado por los vínculos familiares, laborales o políticos, a la hora de explicar la conquista de crecientes niveles de autonomía familiar y comunitaria en lo que es la "Reserva Limitas".

En Limitas, como espacio de vida campesino, confluyen múltiples trayectorias personales y colectivas a partir de lazos familiares, laborales y políticos. En todos los casos se trata de una experiencia vital de des-subjetivación: dejar de ser jornalero, dejar de ser colono o productor algodonero. Pero a la vez, a este corrimiento se le conjuga otro, de modo que se asiste a un doble desplazamiento en las posiciones materiales y simbólicas de las poblaciones que habitan este territorio. El hecho de des-sujetarse de posiciones e identidades anteriores en todos los casos significó también recrear la propia existencia. Se asumieron a sí mismos como comunidad de productores directos, que detenta el control de un área geográfica especifica, territorializándose en la figura de la "Reserva de Limitas".

Acá yo ahora lo que encuentro es que sos peón y sos patrón. Todo al mismo tiempo. Pero te cuesta mucho también. Si vos trabajás, trabajás y no trabajás es lo mismo. 
Nosotros estamos acostumbrados y nos hallamos. Y estamos aquerenciados acá como le dicen. Porque vos podés criar una gallina, un chancho, y en el pueblo no vas a tener un chancho, la gallina también. Y acá esta tranquilo, está tu tranquilidad, vos estás contento.

Yo tenía para comer todos los días pero nunca iba a tener la posibilidad de plantar un pedacito de tierra, o sea que no iba a poder tener una vaquita porque yo tenía mi lechera en campo ajeno, en cualquier momento el tipo vendía y la vaca la tenía que sacar a la calle, y así fue. Y otro de que así tenía en el campo del hermano, hipotecó el hermano en el banco para sacar en esos tiempos donde trabajaba fuerte y después no pudo salir nunca de la cuenta, ese compró...tuvo que vender el campo y bueno le achicó, no sé si tenía sus cuarenta vacas en tres hectáreas, 4 hectáreas, después quedó patio. Después el otro lo tenía en el campo del padre y eran varios hermanos y en la medida en que iban creciendo los hijos, los sobrinos, iban cargando los animales y bueno se achicó todo. $\mathrm{O}$ sea que se apretaron todos y entonces fue eso más o menos. Todo este es el problema de tierras... Y bueno, nosotros... el fin nuestro era tierra, pronto. (Entrevista colectiva con los pobladores de Limitas, 2010)

En este proceso de autoafirmación, como los grafican los extractos de entrevistas, la población de Limitas viene afrontando en condiciones adversas, tanto los desafíos de la instrumentación de formas colectivas para el manejo productivo, como los conflictos por la tenencia de la tierra, o más específicamente, por los distintos lotes que componen el territorio considerado. Más precisamente el espacio físico y social que corresponde a Limitas es ya resultado de diversos esfuerzos colectivos que los habitantes antiguos y recientes fueron activando. Estos esfuerzos los entendemos tanto en su dimensión política como socioeconómica y cultural, en tanto organizan la acción colectica como la reproducción cotidiana. Es por ello que preferimos interpretarlos como procesos de re-existencia, es decir, cuando y donde se asume la voluntad de poder ser como se quiere ser, cuando y donde se hace presente un "sujeto que se des-sujeta reidentificándose", un movimiento que avanza en la apropiación de un discurso y una política en relación con los otros y con la naturaleza, para reinventar la propia vida, re-existir (Leff, 2010:186).

Desde los años de 1980, cuando el cierre del Ingenio aun no había ocurrido pero ya se trataba de una empresa en crisis, los pobladores se organizaron y buscaron apoyo para encontrar alternativas económicas. El Estado, que una década atrás en los años de 1970 había adquirido el Ingenio, no estaba dando como empleador respuestas apropiadas a las expectativas y necesidades de la población que aun se reconocía como trabajadores del Ingenio. En ese tiempo la principal preocupación pasaba por el fortalecimiento y adquisición de capacidades productivas, para autoconsumo y comercialización. Se realizaban periódicamente talleres y reuniones en la zona, en casas de vecinos y en la capilla de Limitas, y actividades de estímulo a la producción agropecuaria orientada a la alimentación, más que a la caña y al algodón que constituían aún el eje de la configuración agraria del Chaco impulsado incluso por el Estado. Éste fue un primer paso en la 
gestación de una respuesta ante la falta de trabajo desde las estrategias familiares, y en el camino que emprendió la población local identificado como el pasaje de jornalero a pequeño productor. Se hicieron campañas agrícolas, se tendieron alambrados, se forestó para monte nativo, se comenzó con la ganadería. Con estas actividades, en el marco de la reciente organización de base campesina, se instaló con mayor fuerza la apuesta por la permanencia en el lugar y la estructuración de la reproducción comenzó a girar mayormente en torno de la puesta en producción de la tierra. Así, se había creado un contexto más favorable para la decisión de resistir, que tomaron algunos pobladores, cuando el Estado en los años de 1990, dictando el cierre del Ingenio, adoptó medidas para que se despejaran las tierras del Ingenio, incluidas las de Limitas. Incluso cuando el Estado implementó el Plan Arraigo en Limitas, otorgando pequeñas parcelas a las familias, hubo una actitud crítica de los pobladores. Ellos, si bien tenían la necesidad de tierra, sabían que las parcelas entregadas servirían solamente para vivienda y en todo caso significarían la imposibilidad de producir. Estas respuestas del Estado hacia los pobladores de Limitas, se desenvolvieron en un contexto de importantes proyectos gubernamentales que no los tenían en cuenta, y de una fuerte presión empresarial sobre el control efectivo de las distintas zonas de Limitas y sus recursos. Ante estos avances los pobladores lograron resultados variables. Frente a los empresarios y productores de diversas escalas, que explotaban algún recurso de Limitas, se entablaron disputas judicializadas o no. Frente al Estado, la población ahora organizada, además de resistir a los intentos de desalojo pasaron a elaborar contrapropuestas.

De la decisión de permanecer en los años de 1980 se pasó en los años de 1990 a la reivindicación, control y defensa, de las aéreas necesarias para las actividades productivas, sobre todo la ganadería campesinas que ya habían comenzado a desplegar. Esta intencionalidad, que iba reforzando la concreción y armado de la "Reserva de Limitas", se consumó en el día a día con nuevas acciones colectivas y gestiones ante el Estado. En este sentido, los pobladores señalan varios momentos críticos en la consolidación de la Reserva. A principio de los años de 1990 se realizó una movilización y toma de la cámara de legisladores de la provincia de Chaco con el propósito de que se reconozca el derecho a la tierra campesina en un conjunto de zonas que luego serían Reservas de la UNPEPROCH, incluida Limitas. Mientras, se resistió el desmonte compulsivo que algunos empresarios realizaban en el paraje. En esos años, también se vivió con ansiedad el despliegue del proyecto impulsado por el municipio para crear una cooperativa de 400 familias de ex empleados del Ingenio Las Palmas, que explotaría las tierras de Limitas sin la participación de sus habitantes. Según los relatos recogidos, durante el año 2004 se entabló un conflicto con un 
empresario que pretendía apropiarse de parte de las tierras de Limitas, alambrando y ocupando con ganado. La resistencia de los pobladores hizo con que este tuviera que retirarse al año siguiente. Simultáneamente un empleado municipal hizo un intento similar, alambrando en forma privada, tierras de Limitas. Si bien se rechazó esta acción de "usurpación", según indican los campesinos, hubo una reincidencia en el año 2009. Aunque se logró evitar en dos oportunidades que este funcionario y comerciante alambrara, no se pudo impedir que esta persona usufructuara económicamente de los recursos de Limitas. Entre el año 2006 y 2007, un empresario oriundo de La Leonesa, robó ganado e intento desalojar una familia de Limitas. Finalmente, desde el año 2009, las familias que habitan Limitas denuncian las fumigaciones y apropiaciones de tierras que realiza una empresa arrocera en la zona de Limitas. Esto ha traído problemas de salud entre los habitantes. Más allá de estos problemas y conflictos, los pobladores relatan un gran número de episodios menores, pero que componen un escenario de tensión permanente. A partir de 2005 se intensificó el asedio de empresarios ganaderos. En entrevistas colectivas los pobladores de Limitas recordaban: "nos cortaban los alambres y soltaban los animales en medio de las plantaciones". Estos hechos reiterados, por ejemplo, hicieron fracasar un proyecto financiado por el Instituto de Investigaciones Forestales y Agropecuarias (IIFA) para la plantación de algarrobo.

Sin embargo, esta gimnasia de confrontación y defensa del lugar no solo modeló a la organización de base, sino que puso en agenda gubernamental el caso Limitas. En cuanto a la primera cuestión, hablamos de la recuperación económica, ahora en tanto productores directos, vía cultivos de autoconsumo y ganadería. Dicha recuperación siguió un camino que dinamizó la organización comunitaria del espacio con la delimitación de lotes para el pastoreo comunitario (por ejemplo, en el 278), la construcción de corrales comunitarios, o el uso comunitario de herramientas agrícolas, o el cultivo de granos en forma colectiva para el alimento del ganado. En cuanto a la segunda cuestión, las acciones y gestiones para afirmar el control campesino de Limitas, terminaron por conformar una instancia de dialogo con el Estado, que derivó en acuerdos que están en ejecución.

En otras palabras, repensar el proceso de la conformación y consolidación de la "Reserva de Limitas" remite en primer lugar, a un acontecimiento identitario, el cual entendimos como un proceso de des-subjetivación de identidades pre existentes y la conformación de una identidad o de una existencia campesina o de pequeño productor con derecho a reclamar frente a los poderes estatales, a ocupar y a resistir la intrusión en su territorio. En definitiva, más allá de lo que puede sostener la doctrina jurídica, no sólo se trata de sentirse con "derecho a" sino sentirse "dueño de". 
Sin embargo, se trata de ser dueño de una forma particular. Frente al ordenamiento jurídico argentino que sostiene que la única forma de propiedad reconocida es la propiedad privada e individual (para aquellos que no portan una identidad étnica), la Reserva de Limitas se posee, se controla y se maneja de forma comunitaria. Es por ello, que en la articulación de ambos acontecimientos, Limitas se presenta como "un campo de experimentación social" (Santos; 2001).

\section{Tensión entre la emancipación y regulación social como situación de interlegalidad}

Familias en Limitas, eran, en realidad eran 19 familias, lo que pasa es que ya tienen muchos hijos grandes que se quieren independizar, entonces se nos aumentó la lista. Tenemos en el censo, 26 o 28. En 2400 hectáreas. En realidad es una reserva, una reserva, viene a ser nacional otra vez de vuelta, viene a ser nacional pero en beneficio de la gente que va a estar viviendo ahí. Sigue siendo del Estado. Es todo nuevo el tema ahí, o sea nosotros tenemos un pedido de tierra, justamente eso con un proyecto de desarrollo, que incluye luz eléctrica, hacer electrificación rural. No hay nada, o sea está la luz, hay que hacerle los tendidos a los domicilios todo eso, hay gente que era más antiguo si ya le llegó, tienen luz, se lo llevaron el año pasado, pero ahora hay que hacer todo nuevo lo que se está haciendo, se presentó nota... a través de la organización nuestra. Pero ahora se está haciendo un planteo, un proyecto bastante interesante, donde se hace de todo digamos, la infraestructura de vivienda, están ellos con un programa de la Subsecretaría, la Subsecretaría de Tierras, está el gobierno, estamos nosotros como organización. Nada más que esto es comunitario para hacer todo lo que es infraestructura de vivienda, y lo que nosotros hacemos es Reserva de producción digamos. O sea de campo, de todos los lotes. Porque ahí por ejemplo, es una Reserva comunitaria, que la UNPEPROCH como organización social, organización de pequeños productores, exige al Estado provincial y exige al Estado nacional, que el Estado Nacional le venda al Estado provincial, cuando se logra que el Estado nacional dice si yo le voy a vender al Estado provincial había que convencerle y exigirle al Estado provincial que compre, no es que el otro decía si yo compro. Nosotros tampoco podíamos decir bueno no, nosotros vamos por esta vía y le exigimos al Estado provincial que compre las tierras, porque él podía decir si yo compro las tierras y después le doy a Eurnekian. El Estado decía bueno yo no puedo regalar, yo te entiendo a vos pero yo regalo no puedo hacer, eso es lo que te dice la ONABE. Entonces nosotros convencimos a la ONABE que le venda a la provincia, que no le venda al Lirusi (un empresario de la zona) y que no le venda a Eurnekian, ese convencimiento tuvimos que hacer, pero no ir a hablar con ellos y fue el convencimiento, de hacer censos, de cuanta gente, que cantidad de familias, que cuantas cosas, y cuanta tierra necesitábamos y que producción teníamos y de ahí en más se fuerza a que minimamente alguien se haga cargo. Si el Estado nacional no lo puede regalar que lo compre la provincia pero nosotros queremos hacer, y fue así. Y en el 94 se firma, en el 94, 95 se hace todo el despelote y en el 95 se firma el convenio, se legisla que la provincia termina comprando, que en el 2006 se termina de pagar, en realidad no pagan en el 2006 todo, pagan en el 2007 y recién ahora nos están avisando de que está todo pagado. Entonces el documento dice que hay un convenio de las tres partes, nosotros si el Estado provincial, o sea cuando él terminaba de pagar esas tierras tiene que ir el Instituto de Colonización y colonizar las familias que nosotros pedimos. No 
puede venir otro a entrar que no sea en consentimiento de los pobladores y la organización, porque sino el Estado va a decir, bueno sí yo compro ahora total de acá a 10 años le vendo a Eurnekian. Y nosotros podemos, o cualquiera de las dos cosas se puede hacer rescindir ese contrato. Si la organización no está de acuerdo cómo se está implementando nosotros hacemos rescindir ese contrato. Ahí recién el Estado nacional dice si la provincia hace esto. ¿Qué hacemos nosotros? Le sacamos y vamos por fuera, pero hoy por hoy tenemos que ir por ahí. ¿Entendes? Si esto se cumple y se cumplió, gracias a dios se cumplió, que se pagó todo ahora. Hablábamos con Capitanich hace poquito, que es el gobernador de acá, me dijo que está todo pagado y que nos tenemos que juntar a armar un consejo de o una forma para armar, a ver como hacemos, empezar a charlar como hacemos esto, si va a quedar en comunitario general para siempre, un título comunitario ¿En beneficio de quien, para quien, cómo? O vamos a parcelar y que cada cual, eso tenemos que trabajarlo. El convenio digamos, dice 'debe estar asociado', estar minimamente, o sea todo lo que es más o menos una parte del párrafo que el estatuto pide, lo implementan en el convenio para que sea legal digamos. Porque sino es cómo te digo, la provincia hoy me puede decir sí yo compro, pero el tema es que si yo compro, y compro por comprar porque el Estado me dice que compre está todo bien, ellos pueden hacer sus negocios otra vez. Entonces lo que nosotros pedimos es que ahí dice, 'La Unión de Pequeños Productores', es cómo que, no me acuerdo bien como está armado digamos, pero sí, la figura es la Unión de Pequeños Productores que pide en beneficio de los asociados esas tierras. Y viene y dice que no puede entrar otro que no sea a través de la organización, pero no quita que la tierra esté, hoy por hoy está todo ocupado, lamentablemente a nosotros siempre nos sigue faltando tierra, como dijo el Dr. Legal ustedes nunca van a terminar de solucionar porque la demanda es todos los días. Fíjate, después de ésta ocupación de tierras y después de lo que sale Limitas, que esto acá fue un comentario internacional más o menos por todos los quilombos que tuvimos, policía, gendarmería todo, después que se gana esto sabes qué, una lluvia de llamadas que hay miles de problemas. Mira de Laguna Limpia, nosotros no tenemos socios en Laguna Limpia, y nos llamaron una familia que le estaban por desalojar, y yo el otro día a la mañana estuve ahí, estuvimos, llevamos al abogado. (Entrevista, sobre situación políticoadministrativa y legal de Limitas, a Oscar, presidente UNPEPROCH, 2009)

La extensa cita da cuenta del proceso que fue gestando y encausando a la Reserva de Limitas en un contexto adverso en cuanto a la política pública y a la presión empresarial por la tierra, pero en paralelo a la conformación y consolidación de la organización en la provincia. Así, garantizar los acontecimientos que se venían desarrollando en Limitas, tal cual los hemos definido, implicaba una determinada estrategia política que tuviera la capacidad de entablar diálogo con los poderes estatales pero que a su vez se mantenga fiel con las apuestas políticas de la organización.

En este sentido, la territorialización de los acontecimientos se expresa como una particular forma de ocupación. En términos generales, la organización ha logrado a través de la ocupación de tierras ${ }^{4}$, la entrega de tierra pública para sus asociados (GEPCyD; 2009). Siguiendo a Mançano

\footnotetext{
4 "Acá hay familias viviendo desde hace cuarenta años, entonces cuando a éstas familias las quieren desalojar es donde se levanta la organización en defensa de ellos y en defensa de ellos viene un grupo más a acompañarlos, pero la causa es a los pobladores de origen y los otros al venir a acompañar y pedir permiso
} 
Fernandes (2008), consideramos a la ocupación "como forma de acceso a las tierras es una acción de resistencia inherente a la formación del campesinado dentro del proceso contradictorio del desarrollo capitalista" (pág. 235). Pero además, en la medida en que la ocupación de tierras se debate entre la legalidad y la ilegalidad, entre lo justo y lo injusto, para el campesinado se transforma, como podría sostener Gargarella (2005), en un derecho a resistir al derecho. En este caso, se resiste nada menos que el derecho de propiedad con el objetivo de garantizar el derecho a la vida.

Sin embargo, las Reservas son resultado del proceso de diálogo y disputas con el poder legislativo y ejecutivo provincial, conformándose en un instrumento jurídico -político administrativo que está marcado por la tensión entre la emancipación y la regulación. La Reserva adquiere un carácter emancipatorio, en primer lugar, de las lógicas del mercado donde el acceso a la tierra se define a través de relaciones comerciales de compra-venta y por ende, realza el valor social que tiene la tierra para el campesinado en detrimento del valor económico asignado por el mercado.

En segundo lugar, en el caso concreto de la Reserva de Limitas, la apropiación del territorio en forma comunitaria supone la revalorización y recreación de concepciones y prácticas subalternas que fueron suprimidas o marginalizadas por las concepciones liberales que dominaron en la conformación de los principales ordenamientos jurídicos en Argentina. En efecto, el proyecto político de la nación argentina que se plasmó en la Constitución Nacional de 1853 y en el Código Civil Argentino en vigencia (con modificaciones) desde 1871 imprimió un sentido de propiedad delimitada como un dominio exclusivo y perpetuo en el sentido de la tradición romana, pero, por sobre todo, al receptar el modelo de propiedad del liberalismo, adquirió un carácter exclusivamente individual.

En este contexto, el reconocimiento de la propiedad comunitaria para poblaciones no indígenas sigue siendo un reto para la UNPEPROCH como así también para otras organizaciones

para acampar, para estar y para producir, dejan de ser usurpación. Por eso nosotros la lucha que le damos, muchos dicen no sé que hace la UNPEPROCH porque la UNPEPROCH siempre consigue. Hoy por hoy están las Reservas, en realidad la idea es después que se pueblen y que vengan, ya por lo menos cuando ya tienen garantizado que están pudiendo producir." (Entrevista a dirigente provincial de UNPEPROCH). La cita nos permite sostener que cuando hacemos referencia al término de ocupación, por un lado, lo distinguimos del término jurídico de usurpación porque consideramos que no se trata de un delito en la medida en que es una acción pública y pacífica. Por el otro, hacemos referencia a un término sociológico en tanto implica la dominación material y la apropiación simbólica de un espacio geográfico. 
campesinas del país. Es aquí donde lo comunitario como acontecimiento adquiere su verdadera dimensión, ya que pone de relieve la existencia de ordenamientos jurídicos no estatales, que en el caso de Limitas dan cuenta de la existencia de situaciones de interlegalidad, es decir, situaciones donde se superponen, articulan, e interpenetran varios espacios jurídicos mezclados que terminan configurando un derecho poroso constituido por múltiples redes de órdenes jurídicos, signados por intersecciones de diferentes líneas de fronteras (Santos; 2001).

Es en este punto donde la Reserva como figura jurídico-política administrativa marca su revés, es decir, su carácter regulatorio. Si bien, por un lado, el gobierno provincial "reserva" a nombre de los asociados de la UNPEPROCH un determinado predio, por el otro, también se "reserva" para sí la titularidad dominial del mismo. Es cierto que dicha situación en Limitas puede analizarse desde dos puntos de vista: por un lado, puede pensarse que no se han alcanzado los tiempos políticos para el traspaso de la titularidad dominial. Bajo esta hipótesis la organización y los pobladores no estarían reclamando el traspaso de la titularidad sobre el territorio controlado, ya que no existe una legislación ni voluntad política para alcanzar un derecho de propiedad comunitario. Por el otro lado, la segunda hipótesis a construir, nos sitúa en el recelo de los poderes estatales hacia la capacidad productiva del campesinado en espacios geográficos que, con las transformaciones del agro chaqueño, se han valorizado de forma considerable.

En este contexto general de falta de reconocimiento de una juridicidad comunitaria y de amenazas de despojo, para el caso Limitas, la estrategia de la organización es avanzar sobre el control comunitario del territorio (como por ejemplo, con el asentamiento de los compañeros/as de San Martín) pero también a través del fortalecimiento de las condiciones de vida y los aspectos productivos, siempre en consonancia con un diálogo con el gobierno nacional y el provincial. En este recorrido, signado por el pulso de la disputa territorial, surge el fideicomiso como política pública. En Limitas, la particularidad de esta figura legal, reside en que con ella se resolvió al menos temporalmente la fricción que se produce entre las concepciones y actuaciones del derecho según el Estado o la organización campesina. El fideicomiso pareciera venir a resolver la situación de interlegalidad puesta de manifiesto en la interfase socio jurídica que plantea Limitas.

Sin embargo, el fideicomiso parte de un diagnóstico común a otras políticas públicas -tanto nacionales como provinciales- donde el campesinado es definido a partir de sus carencias estructurales: la ausencia de niveles de capitalización, producción para la subsistencia, el carácter marginal de su producción, etcétera (véase al respecto, Barbetta, Domínguez y Sabatino; 2012) para a partir de ahí generar una serie de transformaciones a nivel productivo, de infraestructura y 
habitacional, a saber: a) en cuanto a la producción animal, mejorar la "eficiencia" de producción a través de la infraestructura, la alimentación, la sanidad, el manejo y la genética adecuada; b) en lo relativo a la producción agrícola, "mejorar los rendimientos" a través de la incorporación de infraestructura, maquinaria y herramientas apropiadas y practicas adecuadas de manejo de los recursos como rotación de cultivos, uso de abonos orgánicos y abonos verdes, recuperación de suelos degradados, etc.; c) en torno a la comercialización, lograr la comercialización en conjunto de todos los productores del paraje, a través del trabajo organizativo, la planificación colectiva de la producción, la infraestructura necesaria para el acopio, la creación de puntos de venta directos en los pueblos y canales de comercialización, la gestión de la marca colectiva, etc. y por último, d) generar valor agregado en origen mediante la construcción de plantas de procesamiento de la producción primaria, tanto agrícola como ganadera (Informe Limitas, Secretaria de Desarrollo Rural y Agricultura Familiar de la Nación, Chaco).

En Limitas, la puesta en marcha de los servicios del consorcio rural supone un antecedente desde una perspectiva de desarrollo rural que una intervención estatal que busca "que modifiquen el atraso y marginación de los pequeños productores" (Ley del Chaco, 6547/10).

Así el fideicomiso, al igual que los consorcios rurales, se presenta como una herramienta de inclusión en el contrato social en base a la incorporación de infraestructura pero también de conocimientos con el objetivo de mejorar la eficiencia y por ende, los rendimientos. En otras palabras, supone la intención del Estado por tutelar tanto a los territorios como a los sujetos que lo controlan, para lograr la adopción de una racionalidad que suponga su inclusión en la estructura básica del sistema económico. Por su parte, para la organización supone, aún a costa de reconocer al Estado nacional como titular registral de los lotes que conforman la Reserva, neutralizar la presión sobre la tierra por parte del empresariado rural y poder desplegar formas comunitarias de acceso y uso efectivo de un conjunto significativo de bienes naturales.

\section{A MODO DE REFLEXIÓN FINAL}

A lo largo del artículo intentamos dar cuenta de la Reserva de Limitas como una figura jurídico política administrativa que, como caso único, nos permite pensar situaciones de interlegalidad entre la acción política de la organización pero en relación de diálogo y disputa con los poderes estatales. En ella se tensiona tanto la emancipación social como la regulación en un territorio determinado. En este sentido, Limitas se presenta como una situación de interlegalidad en la medida en que se tensionan diferentes ordenes jurídicos en un contexto de disputas por 
definir el acceso, las formas de tenencia y uso de la tierra, y (in)visibilizan sentidos de derecho. Por un lado, el Estado se "reserva" la titularidad de las tierras pero al mismo tiempo, a través de una dotación de recursos, reconoce el derecho de los pobladores a habitar y producir en ese espacio geográfico a fin de desplegar una territorialidad campesina. Por el otro, hemos dado cuenta de una lógica política de la organización que se ha plasmado en conjunto de acontecimientos que si bien cede al Estado derechos en torno a la tenencia de la tierra, busca consolidar la presencia campesina y su reproducción material y simbólica como forma de ser fiel a la organización comunitaria del territorio.

\section{BIBLIGRAFÍA}

ADÁMOLI, Jorge; TORRELLA, Sebastián A. y GINZBURG, Rubén. La Expansión de la Frontera Agrícola en la Región Chaqueña: Perspectivas y Riesgos Ambientales, en Agro y Ambiente: una agenda compartida para el desarrollo sustentable. Buenos Aires, Foro de la Cadena Agroindustrial Argentina. 2010

BADIOU, Alan El Ser y el acontecimiento, Buenos Aires, Editorial Manantiales, 1999.

BARBETTA, Pablo, DOMINGUEZ, Diego y SABATINO, Pablo. La ausencia campesina en la Argentina como producción científica y enfoque de intervención. Revista Mundo Agrario - De Estudios Rurales, Centro de Historia Argentina y Americana (IdIHCS - UNLP - CONICET), № 25, segundo semestre 2012

BERGAILO, Graciela Elizabeth Danza en el viento. Memoria y resistencia Qom (toba), Segundo Premio "Ramón de las Mercedes Tissera" al Ensayo 2002, Subsecretaría de Cultura de la Provincia del Chaco, 2002.

DAL PONT, Silvina y ORDOQUI,_María Soledad. Caracterización económica de la provincia de Chaco, disponible en http: //www.agro.uba.ar/apuntes/no_4/chaco.htm, 2008

GRUPO DE ECOLOGIA POLITIICA COMUNIDADES Y DERECHOS (GEPCYD) Territorios en Construcción. Transformaciones en la Estructura Productiva y alternativas campesinas en Chaco, ponencia presentada en VI Jornadas de Investigación y debate, "Territorio, poder e identidad en el agro argentino", Instituto de Investigaciones Neohistóricas- CONICET, Resistencia, Chaco. Mayo, 2009.

GIARRACCA, Norma y TEUBAL, Miguel. Del desarrollo agroindustrial a la expansión del 'agronegocio': el caso argentino", en Mançano Fernández, B. (Coord.,) Campesinado y Agronegocios en América Latina, Buenos Aires, CLACSO-ASDI, 2008.

GIGENA, ANDREA ET.AL. Relevamiento y sistematización de problemas de tierra de los agricultores familiares en la Argentina. 1a ed. Buenos Aires: Ministerio de Agricultura, Ganadería y Pesca de la Nación, 2013. 
GRUPO DE ESTUDIOS RURALES. Desalojos y arrinconamientos de campesinos y comunidades indígenas en la Argentina, Realidad Económica № 203, abril-mayo, 2004.

LEFF, Enrique. El desvanecimiento del sujeto y la reinvención de las identidades colectivas en la era de la complejidad ambiental”. Polis, Revista de la Universidad Bolivariana, Volumen 9, № 27, Chile, 2010.

MANÇANO FERNANDES, Bernardo. La ocupación como una forma de acceso a la tierra en Brasil: una contribución teórica y metodológica, en Sam Moyo y Paris Yeros [coord.] Recuperando la tierra. El resurgimiento de movimientos rurales en África, Asia y América Latina. Buenos Aires: CLACSO, Consejo Latinoamericano de Ciencias Sociales, 2008.

MARI, Óscar Ernesto. La transición entre dos ciclos y sus efectos sociales en un territorio argentino Conflictos de convivencia en el Chaco ante una nueva etapa colonizadora (1920-1940), Revista de Geografía Norte Grande, no 42, 2009.

SANTOS, Boaventura de Sousa. A crítica da razão indolente. Contra o desperdício da experiência, Brasil, Cortez Editora, 2001.

SLUTZKY Daniel. Estructura social agraria y agroindustrial del Nordeste de la argentina: Desde la incorporación a la economia nacional al actual subdesarrollo concentrador y excluyente, disponible en http://www.iade.org.ar/uploads/c87bbfe5-1e95-22be.pdf; 2011

Trabalho enviado em 11 de agosto de 2014.

Aceito em 22 de setembro de 2014. 